\title{
Predicting and Preventing Sudden Cardiac Death in the Young
}

\author{
Erika Maharani \\ Department of Cardiology and Vascular Medicine, Faculty of Medicine, Public Health and Nursing, Universitas Gadjah Mada - Dr. \\ Sardjito General Hospital, Yogyakarta, Indonesia
}

\author{
Corresponding author: \\ Erika Maharani, MD, - email: erikamaharyo@yahoo.com \\ Department of Cardiology and Vascular Medicine,Faculty of Medicine, Public Health and Nursing, Universitas Gadjah Mada - Dr. \\ Sardjito Hospital \\ Jalan Farmako Sekip Utara, Yogyakarta, Indonesia 55281
}

\begin{abstract}
Sudden cardiac death (SCD) is an unexpected death condition and occurs with unknown causes or cardiac condition. The SCD incidence at young age is a rare condition but when this condition happens it become traumatic event for both family and community. Incidence rate of death on 1-35 years old is around $0.8-2.8 / 100.000$ each year. This number also varies according to age and sex. Death incidence on 1-4 years old is more common than on 5-10 years old group. This number is increasing after 15 years old, where age of 31-35 years has 10 fold risk of SCD compared to 1-10 years old group. Ratio of SCD in men and woman are 2: 1. Early detection of underlying heart disease is the most important action of preventio. If young people are known to have a risk of SCD, the lifestyle changing and appropriate medical therapy, including implantation of implantable cardioverter defibrillator, according to risk stratification must be done.
\end{abstract}

\section{Etiology}

The underlying mechanism of sudden cardiac death (SCD) with normal heart is still not fully understood. In $50 \%$ of cases with a normal heart structure, SCD is the first manifestation of an illness. ${ }^{1}$ The combination of precipitating factors and susceptible myocardium may be the initial factors that underlie lethal arrhythmias that cause in SCD. ${ }^{1}$

Cardiac abnormalities that underlie SCD at a young age individual including congenital heart disease, coronary artery abnormalities, myocarditis, hypertrophic cardiomyopathy, dilated cardiomyopathy, left ventricular non-compaction cardiomyopathy, arrhythmogenic right ventricular cardiomyopathy (ARVC) and electrical channel disturbances (channelopathies) such as long QT syndrome (LQTS), catecholaminergic polymorphic ventricular tachycardia (CPVT) and Brugada syndrome. . $3,4^{2,4}$

Coronary heart disease is still the main cause cardiac abnormality in the older age group, while abnormalities of electrical channel cardiomyopathy or channelopathies are more common at a young age individual. An autopsy is not always performed in individual who experiences SCD, so there is often bias in identifyng incidents and determining the cause of death. Several methods have been investigated to reduce and prevent SCD in population, one of which is by electrocardiograms (ECG) screening in certain populations such as in athletes. ${ }^{5}$ 


\section{Prodormal Symptom and Warning Sign}

Identification of prodormal symptom and clinical signs in SCD group is very important. Predictive ability can be used as a reference in conducting interventions such as diagnostic screening, primary prevention or implantation of ICD. Symptoms which arise will be associated with underlying heart disease condition. ${ }^{6}$ Symptoms such as chest pain, palpitations and shortness of breath are not specific. Syncope and presyncope become one of the most frequent symptoms that precede cardiac arrest incidence. ${ }^{5}$ Other prodormal symptoms are that associated with sudden cardiac arrest are non-sustained arrhythmias that accompanied by palpitations, chest pain, syncope and seizures. This condition demonstrates fluctuations of blood pressure and pulse pressure. Risk of SCD will increase if those symptoms appear accompanied by cardiac abnormalities. ${ }^{6}$

An aggressive diagnosis is needed to determine heart abnormalities before SCD occurs. An SCD becomes high risk marker of future cardiac arrest. Symptoms such as chest pain, palpitations, shortness of breath, presyncope and syncope are not typical but these complaints can be used as a warning sign of a dangerous heart abnormality. ${ }^{5}$ Evaluation of overall heart condition needs to be done immediately in young populations who experience chest pain during exercise that is not affected by movement, inspiration, or palpation and without a clear non-cardiac cause, especially in patients at high risk of cardiac abnormalities, a history of sudden death in the family, or syncope during training that is preceded by palpitations. $^{7}$

\section{Screening}

An SCD often becomes first symptom that occurs and underlies heart abnormalities at a young age, therefore clear concept of cardiovascular screening is needed. Screening can recognize or increase suspicion of cardiac abnormalities in people that previously not been known of cardiac abnormalities. It can reduce the incidence of SCD in asymptomatic individual. Screening will give a time to SCD prevention interventions in risk group population. Prevention actions that can be performed include avoiding excessive physical activity or competitive sports, appropriate medication according to heart disorders (e.g. beta blockers in LQTS and CPVT) and primary prevention with ICD placement in high-risk individual. Screening programs can also be targeted at certain populations or subgroups, for example in athletes. However, SCD can appear at rest or during mild activity. ${ }^{5}$

History of disease and physical examination are less effective in identifying risk groups. Therefore, use of standard 12 leads electrocardiography (ECG) can provide additional screening data to detect underlying heart abnormalities. Resting electrocardiography can assess several etiologies of sudden cardiac death such as HCM, LQTS, Brugada syndrome, ARVC and Wolf Parkinson's white syndrome. However the positive predictive value (PPV) of ECG in assessing cardiac pathology is quite low. Therefore a number of other effective screening modalities are needed so that they can get rid of cardiovascular abnormalities. For example, $\mathrm{T}$ wave inversion in the right precordial leads of ECG is a typical picture for ARVC, but this picture can be normal in the general 
population and not related to cardiac structural abnormalities. ${ }^{5}$

Some working groups recommend a history of cardiac history and physical examination must to be standard in screening the risk of SCD (especially on athletes). Several studies shown history and physical examination are become screening tools that have clinical value that accompanied by additional test such as echocardiography, ECG and genetic examination. $^{8}$

\section{Risk Stratification and Prevention}

Substrate Identification of cardiac abnormalities is a key in prevention of SCD. However, only a few SCD at a young age was found to have been known to have had a previous heart disorder. If the diagnosis is made with a high risk, specific prevention including ICD monitoring can be done. The use of ICD implantation for primary prevention at a young age is increasing in number. Cardiac electrical abnormalities, cardiomyopathy and congenital heart abnormalities are the most common causes of ICD implantation at a young age as primary prevention purpose. Primary prevention is also aimed at young populations with a decrease in heart function (ejection fraction) of less than 30 $35 \%$. As for secondary prevention, ICD insertion is intended for patients with a history of cardiac arrest, ventricular tachycardia with unstable reversible hemodinamic causes has been excluded. Some conditions such as HCM, ARVC, LQTS, CPVT and Brugada syndrome, prevention can be done with lifestyle modification, medication, device use can be suggested to children and young people. ${ }^{5}$

Patients who identified a high risk group for cardiac arrest, ICD implantion should be considered as primary prevention. An ICD implantation in adults has been shown to provide benefits in patients with ischemic cardiomyopathy and left ventricular dysfunction. While for children, the benefits of ICD implantation are still unclear. Implanted ICD in children sometimes provides complications related to fitting with the devices, contain inappropriate shock, malfunction leads and technical difficulties in ICD implantation. On the other hand, ICD implantation is still needed as a primary prevention specifically for the incidence of cardiac arrest due to cardiac abnormalities and arrhythmias due to cardiomyopathy. ${ }^{6}$

\section{Conclusion}

Sudden cardiac death rarely occurs at a young age. In some conditions this can be prevented by screening on high risk populations. The prodromal symptoms, history of SCD in family at a young age, or high risk behavior can increase alertness for the possibility of SCD. Family risk must be searched especially in cases that clearly caused by genetic mutations, such that the risk stratification must be performed.

\section{References}

1. Chugh S.S., Kelly K.L., Titus J.L. 2000. Sudden cardiac death with apparently normal heart. Circulation, 102:649-654.

2. Winkel B.G., Holst A.G., Theilade J., Kristensen I.B., Thomsen J.L., Ottesen G.L., et al. 2011. Nationwide study of sudden cardiac death in persons aged 1-35 years. Eur Heart J, 32:983-999.

3. Bagnall R.D., Weintraub R.G., Ingles J., Duflou J., Yeates L., Lam L., et al. 2016. A prospective study of sudden cardiac death among 
children and young adults. New Engl J Med, 374:2441-2452.

4. Meyer L., Stubbs B., Fahrenbruch C., Maeda C., Harmon K., Eisenberg M., et al. 2012. Incidence, causes, and survival trends from cardiovascular-related sudden cardiac arrest in children and young adults 0 to 35 years of age: a 30 year review. Circulation, 26:13631372.

5. Aro A.L., Chugh S.S. 2017. Prevention of sudden cardiac death in children and young adults. Prog Pediatr Cardiol, 45:37-42.
6. Ackerman M., Atkins D., Triedman J.K. 2016. Sudden cardiac death in the young. Circulation, 133:10061026.

7. Liberthson R.R. 1996. Sudden death from cardiac causes in children and young adults. $\mathrm{N}$ Engl $\mathrm{J}$ Med, 334:1039-1044.

8. Kaltman J.R., Thompson P.D., lantos J., Berul C.I., Botkin J., Cohen J.T. et al. 2011. Screening for sudden cardiac death in the young report from a National Heart, Lung, and Blood Institute Working Group. Circulation, 123:1911-1918. 